\title{
Aspectos biológicos de Leucothyreus dorsalis Blanchard (Coleoptera, Scarabaeidae, Rutelinae)
}

\author{
Sérgio Roberto Rodrigues ${ }^{1}$, Anderson Puker $^{1} \&$ Elison Floriano Tiago ${ }^{1}$
} ${ }^{1}$ Universidade Estadual de Mato Grosso do Sul, Rodovia Aquidauana/CERA km 12, 79200-000 Aquidauana-MS, Brasil. sergio@uems.br, pukeragro@
yahoo.com.br, elisonfloriano@yahoo.com.br

\begin{abstract}
Biological aspects of Leucothyreus dorsalis Blanchard (Coleoptera, Scarabaeidae, Rutelinae). In Brazil there is a shortage of information on bioecology of most species of Scarabaeidae. The aim of this work was to study the biological aspects of Leucothyreus dorsalis Blanchard, 1850 in laboratory and field. The population dynamics of adults was evaluated from January, 2006, to December, 2007, with the use of light traps. Adults collected in the field were kept in plastic recipients containing soil and seedlings of Brachiaria decumbens Stapf, to obtain eggs and start the studies. All developmental stages were monitored and adults and immatures measured to get biometric data. 3,607 adults were collected and the population peaks occurred in November, 2006 and October, 2007, with averages of 145 e 241 individuals collected, respectively. The embryonic period took in the average 15.5 days, the $1^{\text {st }}$ instar 32.4 days, the $2^{\text {nd }}$ instar 38.9 days, the $3^{\text {rd }}$ instar 52.7 days, the pre-pupa 130.7 days, the pupa 23.5 days and the longevity of adults 18 days. The biological cycle was completed in 273.5 days, characterizing the specie as univoltine. From the first to the third instar it was observed an increasing of 4.5 times in the extension and 3.5 times in the width of the larvae. There was an increase of 53.1 times in the larval weight from the first to the third instar. The female pupae were significantly larger and heavier than the male ones. The adult females are larger than the adult males, however, they have similar weight.
\end{abstract}

KEYWORDS. Biological cycle; population fluctuation; rhizophagous pests; Scarabaeoidea; white grubs.

\begin{abstract}
RESUMO. Aspectos biológicos de Leucothyreus dorsalis Blanchard, 1850 (Coleoptera, Scarabaeidae, Rutelinae). No Brasil existe uma escassez de informações sobre a bioecologia da maioria das espécies de Scarabaeidae. O objetivo do presente trabalho foi estudar os aspectos biológicos de Leucothyreus dorsalis Blanchard, 1850 em laboratório e a campo. A dinâmica populacional dos adultos foi avaliada de janeiro de 2006 a dezembro de 2007, com uso de armadilha luminosa. Adultos coletados em campo foram mantidos em recipientes de plástico contendo solo e mudas de Brachiaria decumbens Stapf, para obtenção de ovos e dar início aos estudos. Todos os estágios de desenvolvimento foram acompanhados e adultos e imaturos foram mensurados para obtenção de dados biométricos. Foram coletados 3.607 adultos e os picos populacionais de coleta ocorreram em novembro de 2006 e outubro de 2007, com média de 145 e 241 indivíduos coletados, respectivamente. O período embrionário durou em média 15,5 dias, o primeiro instar 32,4 dias, o segundo 38,9 dias, o terceiro 52,7 dias, a fase de pré-pupa 130,7 dias, a fase pupal 23,5 dias e a longevidade 18 dias. O ciclo biológico completou-se em 273,5 dias, o que caracteriza a espécie como univoltina. Verificou-se que do primeiro ao terceiro instar houve um aumento de 4,5 vezes no comprimento e de 3,5 vezes na largura das larvas. Observou-se um aumento de 53,1 vezes no peso larval do primeiro para o terceiro instar. As pupas das fêmeas foram significativamente maiores e mais pesadas que a dos machos. As fêmeas adultas foram maiores que os adultos machos, porém, apresentaram peso semelhante.
\end{abstract}

PALAVRAS-CHAVE. Ciclo biológico; coró; flutuação populacional; pragas rizófagas; Scarabaeoidea.

Os insetos que vivem no solo ou que nele se desenvolvem, pelo menos durante uma fase do seu ciclo, alimentam-se de material orgânico em decomposição ou tecido vegetal vivo. Aqueles que consomem este segundo tipo de alimento podem se tornar pragas de importância econômica (Ávila \& Parra 2004). Dentre esses insetos, destacam-se as espécies fitófagos da família Scarabaeidae, as quais podem causar perdas na produção, devido principalmente ao ataque de suas larvas, que ao se alimentarem das raízes provocam a murcha, amarelecimento e posterior morte das plantas atacadas (Ávila \& Pípolo 1992).

Sobre a fauna de escarabeídeos edafícolas ocorrentes no Brasil, Morón (2004) relaciona 1.008 espécies, incluídas nas subfamílias, Cetoniinae, Dynastinae, Melolonthinae e Rutelinae. No Brasil as espécies da subfamília Rutelinae e tribo Geniatini são pouco estudadas e consideradas um grupo diverso, com cerca de 165 espécies distribuídas em nove gêneros, enquanto que na tribo Anomalini são registradas 14 espécies em três gêneros (Morón 2004).
Sobre a subfamília Rutelinae na região Centro-Oeste do Brasil, foi realizado o estudo de ocorrência e biologia de Anomala testaceipennis Blanchard, 1856 que ocorre em áreas de pastagens e culturas (Rodrigues et al. 2008). Para algumas espécies de Anomala Samouelle que ocorrem no México e Japão são encontradas informações, sobre descrição de imaturos, aspectos biológicos e comportamento reprodutivo (Del-Bosque 1998; Micó et al. 2003; Arakaki et al. 2004; Ramirez-Salinas et al. 2004).

O gênero Leucothyreus Macleay é constituído por 164 espécies descritas em vários países (Jameson 2008). Nas várias regiões do Brasil, Morón (2004) relaciona a ocorrência de 83 espécies de Leucothyreus, enquanto que no Equador Paucar-Cabrera (2005) menciona a ocorrência de 12 espécies. Nesse gênero são relacionadas algumas informações sobre descrição de imaturos e aspectos biológicos para L. femoratus Burmeister, 1844 que ocorre na Colômbia (Pardo-Locarno et al. 2006). Pardo-Locarno et al. (2005) consideram dentro do complexo de Scarabaeidae pragas em sistemas agroecológicos 
na Colômbia, que os gêneros Plectris, Astaena, Leucothyreus e Macrodactylus são pouco conhecidos e pragas de raízes de plantas: em algumas situações, algumas espécies atingem níveis populacionais superiores a representantes do gênero Phyllophaga Harris.

Poucas são as informações sobre os Rutelinae no Brasil, desta forma o presente trabalho teve como objetivo conhecer os aspectos relacionados à biologia de Leucothyreus dorsalis.

\section{MATERIAL E MÉTODOS}

O trabalho foi desenvolvido na Universidade Estadual de Mato Grosso do Sul (UEMS), no campus da Unidade Universitária de Aquidauana, MS ( $\left.20^{\circ} 28^{\prime} \mathrm{S}, 55^{\circ} 48^{\prime} \mathrm{W}\right)$. Para a obtenção de adultos de L. dorsalis, houveram coletas diárias de janeiro de 2006 a dezembro de 2007, com uma armadilha luminosa com lâmpada fluorescente de 20 watts, modelo Luiz de Queiroz, entre área de pastagem e de culturas (ex. milho, feijão e algodão) na fazenda experimental da UEMS. A armadilha era ligada às $18 \mathrm{~h} 00$ e recolhida às $6 \mathrm{~h} 00$ do dia seguinte. Esse tipo de armadilha tem sido utilizada na coleta de Scarabaeidae devido à atratividade desses insetos por fontes luminosas (Méndez-Aguilar et al. 2005; Buss 2006). Os insetos coletados foram agrupados em semanas para obtenção de média mensal.

Os adultos coletados foram sexados por meio da observação das pernas anteriores, os quais nos machos apresentam os tarsômeros dilatados, semelhante à descrição de Jameson (2008) ao estudar Microchilus Blanchard (Rutelinae), além de dissecação de alguns exemplares para observação da genitália, para confirmação do sexo. De outubro a dezembro de 2007 algumas fêmeas coletadas foram dissecadas para determinar o período de formação dos óvulos, seguindo a metodologia utilizada por Crocker et al. (1999), para espécies de Phyllophaga.

Para verificar a atividade de vôo de L. dorsalis, em 16, 23, 25 a 27 de outubro e de 18 a 21 de dezembro de 2007 foram realizadas vistorias na armadilha luminosa em intervalos de 30 minutos, a partir das $18 \mathrm{~h} 00$ até às $6 \mathrm{~h} 00$. Os dados obtidos foram transformados em $\sqrt{x+1}$, e submetidos à análise de variância e as médias comparadas por meio do teste de Tukey $(\mathrm{P}<0,05)$.

Estudos biológicos foram conduzidos de outubro a dezembro de 2007 a partir de 171 casais de L. dorsalis provenientes de adultos coletados no campo. Os casais foram mantidos em recipientes de plástico $(1.000 \mathrm{ml})$, fechados com tampa plástica contendo furos de 2 a $3 \mathrm{~mm}$. Os recipientes continham solo e mudas de Brachiaria decumbens Stapf cv. Basilisk e, em média dois casais foram acondicionados em cada um. Os recipientes foram vistoriados diariamente, para obtenção de ovos. Os ovos foram transferidos para placas de Petri, contendo solo umedecido e mantidos em câmara climatizada $\left(26 \pm 1^{\circ} \mathrm{C}\right.$ e 12 horas de fotofase). Larvas recém eclodidas eram individualizadas em recipientes de plástico $(500 \mathrm{ml})$, contendo solo e mudas de $B$. decumbens e mantidas nas mesmas condições dos ovos, até atingirem a fase adulta.
As mudas de $B$. decumbens foram substituídas semanalmente por mudas novas, até as larvas atingirem a fase de pré-pupa. Para determinar o número de instares de L. dorsalis, bem como verificar o crescimento larval, em intervalos de 3 a 4 dias, 50 larvas tiveram suas cápsulas cefálicas medidas na maior largura (Rodrigues et al. 2008). O número de instares foi determinado através da regra de Dyar, conforme descrito por Parra \& Haddad (1989).

A biometria de $L$. dorsalis foi realizada mensurando-se o comprimento e largura dos espécimes, além da pesagem dos estágios de desenvolvimento, seguindo metodologia proposta por Silva \& Grützmacher (1996).

Adultos de L. dorsalis foram mensurados, posteriormente mortos e mantidos em estufa de secagem por 48 horas $\left(60 \pm 5{ }^{\circ} \mathrm{C}\right)$ para a pesagem. Os dados biométricos foram submetidos à análise de variância, com as médias comparadas por meio do teste de Tukey $(\mathrm{P}<0,05)$.

As variáveis biológicas estudadas foram, duração do período embrionário, duração do período larval, duração da fase de pupa e longevidade. Esta última variável foi analisada transferindo os adultos recém-emergidos para os recipientes de plástico, contendo solo e mudas de $B$. decumbens efetuandose vistorias diárias.

Espécimes de L. dorsalis foram depositados na coleção "Adolph Hempel" do Instituto Biológico em São Paulo, SP, Brasil e na coleção de insetos do Laboratório de Entomologia da Universidade Estadual de Mato Grosso do Sul em Aquidauana, MS, Brasil. Informações sobre a precipitação foram obtidas da estação meteorológica instalada na UEMS de Aquidauana.

\section{RESULTADOS E DISCUSSÃO}

Coleta de adultos e período reprodutivo.

No primeiro ano de coletas, foram capturados 1809 adultos. De janeiro a março pequena quantidade foi obtida e de abril a agosto os adultos não foram coletados, encontrando-se novamente os espécimes no início de setembro, e de outubro a dezembro obteve-se as maiores médias (Fig. 1). No segundo ano de coletas, foram capturados 1798 adultos. De janeiro a março, novamente pequena quantidade foi obtida, de abril a setembro os adultos não foram coletados, encontrando-se os adultos no início de outubro, sendo novamente de outubro a dezembro obtidas as maiores médias (Fig. 1). Algumas informações sobre a ocorrência de adultos de Leucothyreus são descritas por Castro-Ramírez et al. (2005), Pardo-Locarno et al. (2005), Neita et al. (2006) e Ronqui \& Lopes (2006).

O início da revoada de L. dorsalis em campo, parece estar associada à ocorrência de chuvas. Em setembro de 2006, após ter ocorrido um acúmulo de 51,6 $\mathrm{mm}$ de precipitação os adultos começaram a ser coletados, e no ano seguinte não houve precipitação em setembro e adultos de $L$. dorsalis não foram coletados. Em outubro de 2007 após ter ocorrido um acúmulo de $56,2 \mathrm{~mm}$ de precipitação, novamente os adultos começaram a ser coletados (Fig. 1).

Mondino et al. (1997) estudaram o ciclo de vida de Cyclocephala signaticollis Burmeister, 1847 e verificaram que 


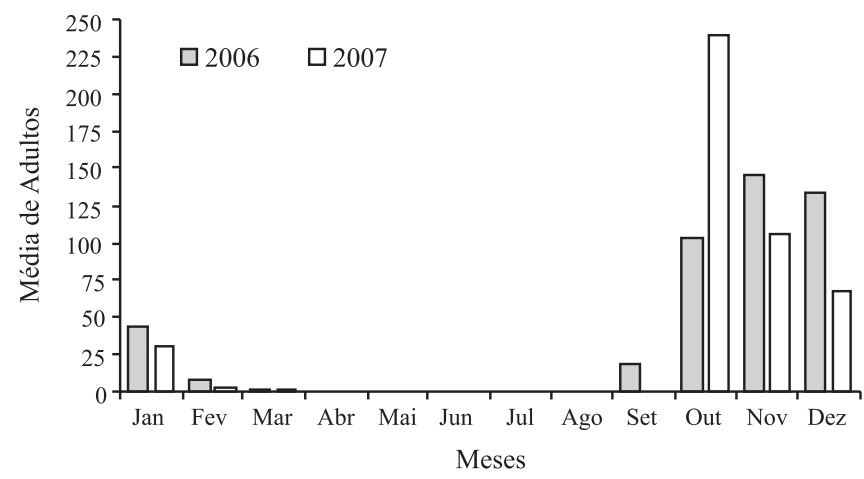

Fig. 1. Quantidade média de adultos/mês de Leucothyreus dorsalis coletados com armadilha luminosa, de janeiro de 2006 a dezembro de 2007 em Aquidauana, MS.

a densidade de adultos é significativamente correlacionada com a umidade do solo, pois cerca de $90 \%$ dos insetos foram obtidos após as precipitações. Para A. flavipennis DelBosque (1998) verificou que a chuva ou irrigação agem como estímulo a emergência. $\mathrm{O}$ efeito da chuva na emergência de algumas espécies de Scarabaeidae, foram também registrados por Gaylor \& Frankie (1979), Potter (1981) e Stone (1986).

Nos dois anos de estudos 3.607 adultos foram coletados, sendo que destes, 2.055 (56,97\%) eram machos e 1.552 $(43,03 \%)$ eram fêmeas havendo uma proporção sexual de 1:1. Segundo Gómez et al. (1999) à proporção sexual dos adultos de Anomala atomogramma Bates, 1888, é de 3:1 entre machos e fêmeas.

Nas fêmeas dissecadas constatou-se a presença de ovos em desenvolvimento cerca de 12 dias após o início da revoada. Em outubro foram encontrados cerca de 5,0 ovos por fêmea, em novembro a média foi de 5,5 ovos e em dezembro foi 2,2 ovos por fêmea. O máximo de ovos encontrados foram 22 e o mínimo de um ovo por fêmea (Tabela I). Em fêmeas de A. testaceipennis, Rodrigues et al. (2008) encontraram ovos durante os meses em que os adultos realizam as revoadas, registrando a maior média em julho.

Adultos de L. dorsalis foram coletados a partir das $19 \mathrm{~h} 00$ e revoaram até às $05 \mathrm{~h} 30$ (Fig. 2). O horário em que foram encontradas as maiores quantidades de adultos no campo, foi entre as $20 \mathrm{~h} 00$ e $20 \mathrm{~h} 30$, entretanto, não houve diferença estatística entre as médias de coletas obtidas entre as $19 \mathrm{~h} 30 \mathrm{e}$ $23 \mathrm{~h} 00$ (Fig. 2). Em Codazzi na Colômbia, Pardo-Locarno et al. (2006) observaram que os adultos de L. femoratus possuem hábito crepuscular. Rodrigues et al. (2008) estudaram o horário da atividade de vôo de $A$. testaceipennis e encontraram que os adultos iniciam a revoada a partir das $18 \mathrm{~h} 30$ e encerram

Tabela I. Média ( \pm EP) de ovos encontrados em fêmeas de Leucothyreus dorsalis, de outubro a dezembro de 2007, em Aquidauana, MS.

\begin{tabular}{ccccc}
\hline Meses & Média \pm EP & N & Amplitude & Total $^{1}$ \\
\hline Out & $5,0 \pm 0,22$ & 195 & $01-19$ & 979 \\
Nov & $5,5 \pm 1,11$ & 21 & $01-22$ & 116 \\
Dez & $2,2 \pm 0,44$ & 10 & $01-04$ & 22
\end{tabular}

$\mathrm{EP}=$ Erro Padrão.

${ }^{1}$ Total de ovos encontrados.

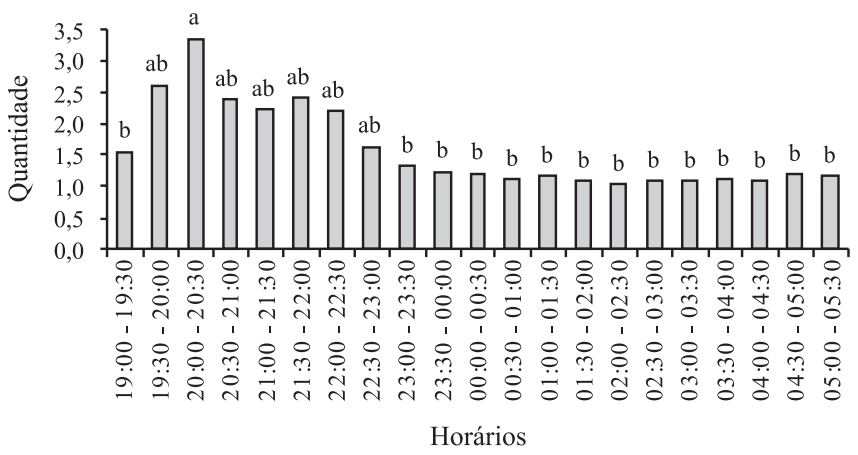

Fig. 2. Quantidade média de adultos de Leucothyreus dorsalis coletados com armadilha luminosa nos diferentes horários de observações. Médias seguidas pelas mesmas letras, não diferem entre si pelo teste de Tukey $(\mathrm{P}<0,05)$, dados transformados em $\sqrt{x+1}$.

as 6 h00 da manhã. Segundo Gómez et al. (1999) os adultos de A. atomogramma estão ativos de abril a junho, revoando entre $20 \mathrm{~h} 00$ e $23 \mathrm{~h} 00$, onde foram encontrados em cópula e se alimentando de folhagens.

\section{Aspectos biológicos.}

Dos adultos de L. dorsalis mantidos nos recipientes de plástico, foram obtidos 262 ovos, sendo que estes foram depositados individualmente em câmaras no solo, as quais, apresentam dimensões internas de 1,9 $\mathrm{mm}$ de comprimento por $1,6 \mathrm{~mm}$ de largura $(\mathrm{n}=78)$. Os ovos apresentaram cor branca e mediram em média $1,8 \mathrm{~mm}$ de comprimento por $1,5 \mathrm{~mm}$ de largura e quando recém-ovipositados pesaram em média 2,3 mg (Tabela II). Segundo Rodrigues et al. (2008) os ovos de A. testaceipennis possuem dimensões de 1,6 $\mathrm{mm}$ de comprimento por 1,0 $\mathrm{mm}$ de largura, sendo que estes apresentam cor branca e também são depositados individualmente no solo.

O período embrionário de $L$. dorsalis durou em média 15,5 dias. O primeiro instar larval durou em média 32,4 dias, enquanto que o segundo e terceiro instar duraram respectivamente, 38,9 e 52,7 dias (Tabela III). A fase de pré-pupa durou 130,7 dias, e esta fase é caracterizada por uma alteração de cor da larva que adquire coloração branca e permanece em uma câmara. O período embrionário de $A$. testaceipennis durou em média 13,2 dias, o primeiro ínstar 26,7 dias, o segundo 19,4 dias, o terceiro 58,2 dias, e a prépupa 50,2 dias (Rodrigues et al. 2008).

As larvas de $L$. dorsalis quando recém-eclodidas apresentaram cerca de 4,2 $\mathrm{mm}$ de comprimento e 1,1 $\mathrm{mm}$ de largura da base do abdome (Tabela II). No segundo estádio, as larvas apresentaram em média $12,1 \mathrm{~mm}$ de comprimento e 2,2 $\mathrm{mm}$ de largura com um aumento de 2,9 vezes para o comprimento e de duas vezes para a largura do primeiro para o segundo instar. As larvas de terceiro instar apresentaram comprimento médio de $18,9 \mathrm{~mm}$ por $3,8 \mathrm{~mm}$ de largura (Tabela II). Do segundo para o terceiro ínstar foi observado um crescimento de 1,6 vez para o comprimento e de 1,7 vez para a largura. Quando compara-se o desenvolvimento larval do primeiro ao terceiro instar, verifica-se que as larvas de $L$. dorsalis aumentaram em cerca de 4,5 vezes seu comprimento 
Tabela II. Médias ( \pm EP) do comprimento, largura e peso das fases de desenvolvimento de Leucothyreus dorsalis, em laboratório $\left(26 \pm 1^{\circ} \mathrm{C}\right.$, fotofase de 12 horas).

\begin{tabular}{|c|c|c|c|c|c|c|c|c|c|}
\hline \multirow[b]{2}{*}{ Fase } & \multicolumn{3}{|c|}{ Comprimento $(\mathrm{mm})$} & \multicolumn{3}{|c|}{ Largura (mm) } & \multicolumn{3}{|c|}{ Peso (mg) } \\
\hline & Média \pm EP & $\mathrm{N}$ & Amplitude & Média \pm EP & $\mathrm{N}$ & Amplitude & Média \pm EP & $\mathrm{N}$ & Amplitude \\
\hline Ovo & $1,8 \pm 0,01$ & 100 & $1,5-2,1$ & $1,5 \pm 0,01$ & 100 & $1,0-1,9$ & $2,3 \pm 0,04$ & 100 & $0,7-3,3$ \\
\hline Larva & & & & & & & & & \\
\hline $1^{\circ}$ ínstar & $4,2 \pm 0,10$ & 100 & $3,5-5,0$ & $1,1 \pm 0,03$ & 100 & $0,9-1,3$ & $3,9 \pm 0,23$ & 100 & $1,3-7,3$ \\
\hline $2^{\circ}$ ínstar & $12,1 \pm 0,28$ & 100 & $8,0-15,0$ & $2,2 \pm 0,06$ & 100 & $1,6-2,9$ & $49,0 \pm 3,16$ & 100 & $13,7-96,7$ \\
\hline $3^{\circ}$ ínstar & $18,9 \pm 0,38$ & 100 & $14,0-23,0$ & $3,8 \pm 0,11$ & 100 & $2,5-4,9$ & $207,0 \pm 14,02$ & 100 & $73,0-330,2$ \\
\hline Pupa & & & & & & & & & \\
\hline Macho & $12,2 \mathrm{a}^{1} \pm 0,21$ & 15 & $10,9-13,6$ & $5,4 \mathrm{a} \pm 0,09$ & 15 & $5,0-5,9$ & $141,1 \mathrm{a}^{1} \pm 8,63$ & 15 & $67,5-189,1$ \\
\hline Fêmea & $13,4 b \pm 0,16$ & 19 & $12,4-14,3$ & $6,1 b \pm 0,09$ & 19 & $5,6-6,8$ & $189,9 b \pm 7,18$ & 19 & $143,4-240,9$ \\
\hline Adulto & & & & & & & & & \\
\hline Macho & $10,1 \mathrm{a} \pm 0,10$ & 50 & $9,0-12,5$ & $4,5 \mathrm{a} \pm 0,03$ & 50 & $3,8-4,9$ & $22,5 \mathrm{a} \pm 1,28$ & 50 & $17,2-31,1$ \\
\hline Fêmea & $11,0 \mathrm{~b} \pm 0,11$ & 50 & $9,5-12,8$ & $4,7 b \pm 0,04$ & 50 & $4,2-5,2$ & $25,0 \mathrm{a} \pm 1,45$ & 50 & $18,2-35,9$ \\
\hline
\end{tabular}

${ }^{1}$ médias seguidas por letras distintas na coluna, diferem entre si pelo teste de Tukey $(\mathrm{P}<0,05)$.

$\mathrm{EP}=$ Erro Padrão.

e de 3,5 vezes sua largura. Pardo-Locarno et al. (2006) encontraram que as larvas de terceiro ínstar de L. femoratus apresentam comprimento variando de 18 a $21 \mathrm{~mm}$, e a largura da base do abdome de 4 a $5 \mathrm{~mm}$.

Com relação ao tamanho das cápsulas cefálicas das larvas de L. dorsalis, foi verificado que a largura da cabeça das larvas de primeiro instar mediu cerca de 1,0 $\mathrm{mm}$ de largura, enquanto que as de segundo e terceiro instar 1,8 e $2,8 \mathrm{~mm}$ respectivamente. A cápsula cefálica das larvas de terceiro instar de L. femoratus apresentam largura variando entre 3 a 3,2 mm (Pardo-Locarno et al. 2006). As cápsulas cefálicas das larvas de terceiro instar de L. dorsalis apresentaram largura inferior às de L. femoratus.

As larvas recém-eclodidas pesaram em média 3,9 mg, enquanto que as larvas de segundo e terceiro instar pesaram respectivamente, 49,0 e 207,0 $\mathrm{mg}$ (Tabela II). Do primeiro para o segundo instar, as larvas de L. dorsalis apresentaram um aumento no ganho de peso de 12,6 vezes, do segundo para o terceiro instar observou-se um aumento de 4,2 vezes. Do primeiro ao terceiro instar larval, observou-se um aumento no ganho de peso de 53,1 vezes.

A duração larval incluindo a pré-pupa durou em média 246,4 dias. A fase pupal L. dorsalis durou em média 23,5 dias (Tabela III). As fases larval e pupal de A. testaceipennis duraram respectivamente 114,7 e 13,6 dias (Rodrigues et al. 2008).

As larvas de L. dorsalis antes de se transformar em pupa construíram com o solo uma câmara pupal, que apresentou dimensões internas médias de $26,1 \mathrm{~mm}$ de comprimento $(17,5-34,2)$ por $8,6 \mathrm{~mm}$ de largura $(6,0-10,7)(\mathrm{n}=09)$. As câmaras pupais, que foram construídas com solo e saliva não eram facilmente desfeitas com a manipulação do solo, o que conferia um ambiente de proteção para a pupa. Segundo Rodrigues et al. (2008) as câmaras pupais de A. testaceipennis medem em média $25 \mathrm{~mm}$ de comprimento por $9 \mathrm{~mm}$ de largura.

Observou-se para as pupas de L. dorsalis que as fêmeas mediram em média 13,4 mm de comprimento por $6,1 \mathrm{~mm}$ de largura e foram estatisticamente maiores que os machos que mediram 12,2 $\mathrm{mm}$ de comprimento por 5,4 $\mathrm{mm}$ de largura
(Tabela II). Além do comprimento e largura, o peso das pupas fêmeas também foi superior ao dos machos, que pesaram respectivamente 189,9 e $141,1 \mathrm{mg}$ e possuíam coloração amarela (Tabela II). As pupas dos machos de L. femoratus possuem corpo ovalado, robusto, coloração amarelo claro e medem cerca de 14 a $16 \mathrm{~mm}$ de comprimento por 7 a $12 \mathrm{~mm}$ de largura (Pardo-Locarno et al. 2006).

A longevidade dos adultos de L. dorsalis foi semelhante para machos e fêmeas, com duração média de 18 dias $(\mathrm{n}=$ 30) (Tabela III). Para A. testaceipennis foi verificado uma longevidade média de 14,1 dias (Rodrigues et al. 2008).

O ciclo biológico de L. dorsalis completou-se em 273,5 dias o que caracteriza a espécie como univoltina (Tabela III). A. testaceipennis completou o ciclo biológico em 139,4 dias (Rodrigues et al. 2008).

Com relação ao tamanho dos adultos, verificou-se que os machos apresentaram 10,1 mm de comprimento por 4,5 $\mathrm{mm}$ de largura e foram estatisticamente menores que as fêmeas que mediram $11,0 \mathrm{~mm}$ de comprimento por 4,7 $\mathrm{mm}$ de largura (Tabela II). Quando compara-se o peso dos adultos, não verifica-se diferença estatística entre machos e fêmeas, que pesaram respectivamente 22,5 e $25,0 \mathrm{mg}$ (Tabela II).

Segundo Ramírez-Salinas \& Castro-Ramírez (2000) os adultos de Anomala inconstans, medem de 14 a $15 \mathrm{~mm}$ de comprimento e cerca de $8 \mathrm{~mm}$ de largura, enquanto que os

Tabela III. Duração média ( \pm EP) das fases de desenvolvimento de Leucothyreus dorsalis, em laboratório $\left(26 \pm 1^{\circ} \mathrm{C}\right.$, fotofase de 12 horas).

\begin{tabular}{lcccc}
\hline Fase & $\begin{array}{c}\text { Duração } \\
\text { (dias) }\end{array}$ & N & Amplitude & $\begin{array}{c}\text { Viabilidade } \\
(\%)\end{array}$ \\
\hline Ovo & $15,5 \pm 0,15$ & 262 & $12-26$ & 93,9 \\
$1^{\circ}$ ínstar & $32,4 \pm 1,27$ & 246 & $12-89$ & 61,0 \\
$2^{\circ}$ ínstar & $38,9 \pm 2,04$ & 150 & $11-147$ & 82,0 \\
$3^{\circ}$ ínstar & $52,7 \pm 2,25$ & 123 & $12-112$ & 88,6 \\
Pré-pupa & $130,7 \pm 6,52$ & 109 & $71-211$ & 31,2 \\
Duração larval & $246,4 \pm 6,23$ & 34 & $183-326$ & 13,8 \\
Pupa & $23,5 \pm 4,96$ & 34 & $08-72$ & 91,2 \\
Adulto & $18,0 \pm 2,18$ & 31 & $05-40$ & 96,8 \\
Ovo a adulto & $273,5 \pm 7,54$ & 31 & $203-337$ & 11,8 \\
\hline
\end{tabular}


adultos de $A$. sticticoptera Blanchard, 1850 medem de 14 a $16 \mathrm{~mm}$ de comprimento por 7 a $8 \mathrm{~mm}$ de largura e os adultos de $A$. denticollis Bates, 1888 medem de 9,3 a 11,0 mm de comprimento por 5,0 a 5,3 $\mathrm{mm}$ de largura.

Agradecimentos. À Universidade Estadual de Mato Grosso do Sul (UEMS) pela concessão da bolsa de iniciação científica ao segundo autor. Ao Dr. Sérgio Ide (Instituto Biológico) pela identificação da espécie estudada.

\section{REFERÊNCIAS}

Arakaki, N.; M. Kishita; A. Nagayama; M. Fukaya; H. Yasui; T. Akino; Y. Hirai \& S. Wakamura. 2004. Precopulatory mate guarding by the male green chafer, Anomala albopilosa sakishimana Nomura (Coleoptera: Scarabaeidae). Applied Journal of Zoology 39: 455-462.

Ávila, C. J. \& J. R. P. Parra. 2004. Influência de fatores físicos edáficos sobre pragas de solo, p. 69-98. In: Salvadori, J. R.; C. J. Ávila \& M. T. B. Silva. (ed.). Pragas de Solo no Brasil. Passo Fundo: Embrapa-CNPT; Dourados: Embrapa-CPAO; Cruz Alta: Fundacep Fecotrigo, 544 p.

Ávila, C. J. \& A. E. Pípolo. 1992. Ocorrência e Danos do "Coró" (Coleoptera: Scarabaeidae - Melolonthinae) em Trigo na Região de Dourados, MS. Anais da Sociedade Entomologica do Brasil 21: 261.

Buss, E. A. 2006. Flight activity and relative abundance of phytophagous scarabs (Coleoptera: Scarabaeoidea) from two locations in Florida. Florida Entomologist 89: 32-40.

Castro-Ramírez, A. E.; H. D. González; V. P. Tabla \& M. A. Morón. 2005. Fauna de melolóntidos (Coleoptera: Scarabaeoidea) asociados al maíz (Zea mays L.) en Los Altos de Chiapas, México. Folia Entomologica Mexicana 44: 339-365.

Crocker, R. L.; W. T. Junior Nailon; J. H. Matis \& R. E. Woodruff. 1999. Temporal Pattern of Ovipositional Readiness in Spring Species of Phyllophaga (Coleoptera: Scarabaeidae) en North Central Texas. Annals of the Entomological Society of America 92: 47-52.

Del-Bosque, L. A. R. 1998. A Sixteen-Year Study on the Bivoltinism of Anomala flavipennis (Coleoptera: Scarabaeidae) in Mexico. Environmental Entomology 27: 248-252.

Gaylor, M. J. \& G. W. Frankie. 1979. The relationship of rainfall to adult flight activity, and of soil moisture to oviposition behavior and eff and first instar survival in Phyllophaga crinita. Environmental Entomology 8: $591-594$.

Gómez, B.; F. J. Villalobos; L. Ruiz \& A. E. Castro. 1999. Observaciones sobre la biologia de melolontidos (Coleoptera: Scarabaeoidea) en una localidad de Los Altos de Chiapas, México. Acta Zoológica Mexicana (nueva serie) 78: 173-177.

Jameson, M. L. 2008. Review of the genus Microchilus Blanchard (Coleoptera: Scarabaeidae: Rutelinae: Geniatini). Insecta Mundi 25: $1-14$.

Méndez-Aguilar, M. J.; A. E. Castro-Ramírez; R. A. Barrantes; C. PachecoFlores \& C. Ramírez-Salinas. 2005. Eficacia de dos tipos de recolecta para registrar la diversidad de melolóntidos nocturnos (Coleoptera: Scarabaeoidea). Acta Zoológica Mexicana (nueva serie) 21: 109-124.
Micó, E.; M. A. Morón \& E. Galante. 2003. New Larval Descriptions and Biology of Some New World Anomalini Beetles (Scarabaeidae: Rutelinae). Annals of the Entomological Society of America 96: 597614.

Mondino, E. A.; A. N. López; H. A. A. Castillo \& D. M. Carmona. 1997. Ciclo de vida de Cyclocephala signaticollis Burmeister, 1847 (Coleoptera: Scarabaeidae: Dynastinae) Y su relación con los factores ambientales. Elytron 11: 145-156.

Morón, M. A. 2004. Melolontídeos edafícolas, p. 133-166. In: Salvadori, J. R.; C. J. Ávila \& M. T. B. Silva. (ed.). Pragas de Solo no Brasil. Passo Fundo: Embrapa-CNPT; Dourados: Embrapa-CPAO; Cruz Alta: Fundacep Fecotrigo, $544 \mathrm{p}$.

Neita, J. C.; J. Orozco \& B. Ratcliffe. 2006. Escarabajos (Scarabaeidae: Pleurosticti) de la selva baja del Bosque Pluvial Tropical "BP-T", Chocó, Colombia. Acta Zoológica Mexicana (nueva serie) 22: 1-32.

Pardo-Locarno, L. C.; J. Montoya-Lerma; A. C. Bellotti \& A. V. Schoonhoven. 2005. Structure and Composition of the White Grub Complex (Coleoptera: Scarabaeidae) in Agroecological Systems of Northern Cauca, Colombia. Florida Entomologist 88: 355-363.

Pardo-Locarno, L. C.; M. A. Morón \& J. Montoya-Lerma. 2006. Descripción de los estados inmaduros de Leucothyreus femoratus Burmeister (Coleoptera: Melolonthidae: Rutelinae: Geniatini) con notas sobre su biología e importancia agrícola en Colombia. Folia Entomologica Mexicana 45: 179-193.

Parra, J. R. P. \& M. L. Haddad. 1989. Determinação do número de instares de insetos. Piracicaba, FEALQ. 49 p.

Paucar-Cabrera, A. 2005. A catalog and distributional analysis of the Rutelinae (Coleoptera: Scarabaeidae) of Ecuador. Zootaxa 948: 1-92.

Potter, D. A. 1981. Seasonal emergence and flight or northern and southern masked chafers in relation to air and soil temperature and rainfall patterns. Environmental Entomology 10: 793-797.

Ramírez-Salinas, C. \& A. E. Castro-Ramírez. 2000. El complejo "gallina ciega" (Coleoptera: Melolonthidae) en el cultivo de maíz, en El Madronal, municipio de Amatenango Del Valle, Chiapas, México. Acta Zoológica Mexicana (nueva serie) 79: 17-41.

Ramírez-Salinas, C.; M. A. Morón \& A. E. Castro-Ramírez. 2004. Descripción de los Estados Inmaduros de Tres Especies de Anomala, Ancognatha y Ligyrus (Coleoptera: Melolonthidae: Rutelinae y Dynastinae) con Observaciones de su Biología. Acta Zoológica Mexicana (nueva serie) 20: $67-82$.

Rodrigues, S. R.; A. Puker; A. R. Abot; C. L. Barbosa; S. Ide \& G. V. Coutinho. 2008. Ocorrência e aspectos biológicos de Anomala testaceipennis Blanchard (Coleoptera, Scarabaeidae). Revista Brasileira de Entomologia 52: 68-71.

Ronqui, D. C. \& J. Lopes. 2006. Composição e diversidade de Scarabaeoidea (Coleoptera) atraídos por armadilha de luz em área rural no norte do Paraná, Brasil. Iheringia, Série Zoologia 96: 103-108.

Silva, M. T. B. \& A. D. Grützmacher. 1996. Biometria de Diloboderus abderus (Sturm) (Coleoptera: Melolonthidae) Coletado em Solo Manejado no Sistema de Plantio Direto. Anais da Sociedade Entomológica do Brasil 25: 377-382.

Stone, J. D. 1986. Time and height of flight of adults of white grubs (Coleoptera: Scarabaeidae) in the southwestern United States. Environmental Entomology 15: 194-197. 